\title{
Fungal pores feel the pull
}

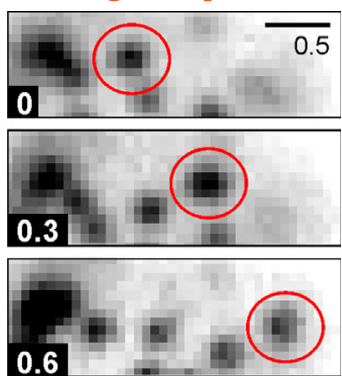

A nuclear pore (red circle) is shown moving in the nuclear membrane of a fungal cell. ing that they rely on a different mechanism to position their pores.

Steinberg et al. found that fungal cells are constantly adjusting the locations of their nuclear pores. In the pathogenic fungus

\section{Chaperones team up twice}
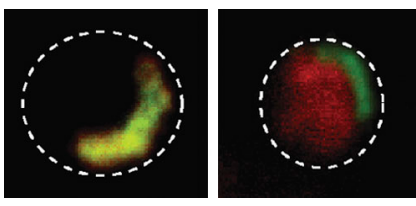

Yeast Hsp100 (red) cozies up to prion fibrils (green) when the cell expresses Hsp70 (left) but not when $\mathrm{Hsp70}$ is inactive (right).

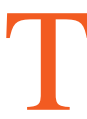

he chaperone Hsp70 lives up to its name, guiding its partner Hsp100 into position in two different situations, Winkler et al. reveal.

Stress can spur proteins to misfold and clump. Hsp70 and Hsp100 enable yeast, bacteria, and plants to restore and reuse aggregated proteins. These chaperones collaborate to pluck proteins from aggregates and feed them through a channel in Hsp100, after which the proteins can refold into their normal shapes. In yeast, the chaperone duo also helps prions spread. Unlike the disease-causing prions of humans, some yeast prions seem to be beneficial, boosting resistance to toxins, for instance. Working together, the chaperones chop prion fibrils into smaller segments that can
Ustilago maydis, the motor proteins kinesin-1 and dynein slide the pores around on microtubules. In budding yeast, by contrast, pore movements required the actin cytoskeleton, suggesting that myosin motors are providing the muscle power.

The team also determined what happened if cells couldn't rearrange their pores. The structures clumped in $U$. maydis cells lacking kinesin- 1 and dynein. Transport of cargoes into and out of the nucleus slowed in cells missing either or both of the motor proteins. When a cell moves its nuclear pores, it also moves its chromosomes. Blocking these movements caused chromosomes to gather at the edge of the nucleus, usually near the pore clusters. This could explain why impeding pore movement inhibits nuclear transport. Misplaced chromosomes might obstruct cargoes traveling into and out of the nucleus.

Steinberg, G., et al. 2012. J. Cell Biol. http://dx.doi.org/10.1083/ jcb.201201087.

move into the daughter cell during division. However, researchers haven't nailed down Hsp70's role during these two processes.

Winkler et al. found that Hsp70 helps direct Hsp100 to clusters of misshapen proteins. For example, the researchers tracked the chaperones in E. coli cells that were lacking either $\mathrm{ClpB}$ (the bacterial Hsp100) or DnaK (the bacterial equivalent of Hsp70). When DnaK was absent, ClpB rarely found its target. But DnaK could still locate and stick to protein aggregates even in the absence of $\mathrm{ClpB}$. The researchers showed that these results also held true for yeast.

The team discovered that the two chaperones have the same relationship during the dismemberment of prion fibrils, with the yeast version of Hsp70 ushering its partner into place. The researchers note that vertebrates don't have an equivalent of Hsp100, but the results suggest that their version of Hsp70 could have a role in prion transmission and pathogenesis.

Winkler, J., et al. 2012. J. Cell Biol. http://dx.doi.org/10.1083/ jcb.201201074.

\section{CLASPing microtubules to the membrane}

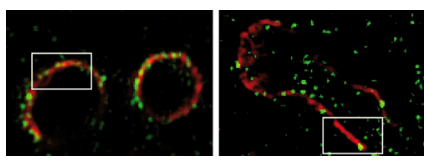

More microtubule ends (green) are located near acetylcholine receptors (red) in a control NMJ (left) than in an NMJ lacking CLASP2 (right). side, opposite the nerve terminal. The protein agrin, which is secreted by the nerve terminal, promotes clustering by stimulating the expression of acetylcholine receptor genes in the muscle nuclei under the synapse and by spurring construction of a scaffold to which the receptors can attach. Schmidt et al. discovered that agrin also prompts receptors to bunch by stabilizing microtubules so that they form a network that points toward the NMJ.
These microtubules spur the addition of acetylcholine receptors to the clusters, the researchers found. For example, cultured muscle myotubes clump their receptors after exposure to agrin. But when the team depolymerized the myotube microtubules with nocodazole, the clusters were $60 \%$ smaller.

Schmidt et al. teased out how agrin affects microtubule behavior. Agrin switches on phosphatidylinositol 3-kinase, which blocks another kinase, glycogen synthase kinase-3 $\beta$. This permits the protein CLASP2 to attach to the side of a microtubule instead of staying at its end, allowing the protein to remain bound to microtubule segments near the cell membrane. The researchers showed that CLASP2 and its partner CLIP-170 help stabilize microtubules and fasten them to the cell membrane where agrin clusters the acetylcholine receptors.

Schmidt, N., et al. 2012. J. Cell Biol. http://dx.doi.org/10.1083/ jcb. 201111130. 